\title{
The distribution of the eigenvalues of Hecke operators
}

\author{
by
}

J. B. Conrey (Stillwater, Okla.), W. Duke (New Brunswick, N.J.) and D. W. FArmer (Lewisburg, Penn.)

\section{Introduction and statement of results. Let}

$$
f(z)=\sum_{n=1}^{\infty} a_{f, n} e(n z)
$$

be a cusp form of weight $k$ for the full modular group. We assume that $f(z)$ is an eigenfunction of all the Hecke operators $T_{n}$, and we set $a_{f}(n)=$ $n^{(1-k) / 2} a_{f, n}$. The assumption that $f(z)$ is a Hecke eigenform gives:

$$
\begin{gathered}
a_{f}(m n)=a_{f}(n) a_{f}(m) \quad \text { if }(m, n)=1 \\
a_{f}\left(p^{j}\right)=a_{f}(p) a_{f}\left(p^{j-1}\right)-a_{f}\left(p^{j-2}\right) \quad \text { for } p \text { prime, } \\
\left|a_{f}(n)\right| \leq d(n),
\end{gathered}
$$

where $d(n)$ is the divisor function. The above relations were first conjectured by Ramanujan based on a computation of the coefficients of the weight 12 cusp form $\Delta(z)=\sum \tau(n) e(n z)$. The two equations were proven for $\tau(n)$ by Mordell, using what are now known as the Hecke operators. The inequality was proven by Deligne as a consequence of his proof of the Weil conjectures. Those results determine everything about $a_{f}(n)$ except for the distribution of the $a_{f}(p) \in[-2,2]$.

Define $\theta_{f}(p) \in[0, \pi]$ by $a_{f}(p)=2 \cos \theta_{f}(p)$. It is conjectured that for each $f$ the $\theta_{f}(p)$ are uniformly distributed with respect to the Sato-Tate measure

$$
\frac{2}{\pi} \sin ^{2} \theta d \theta
$$

This conjecture was first made for cusp forms associated with non-CM elliptic curves, and was extended to Hecke eigenforms for the full modular group by Serre [Ser]. This conjecture appears to be quite deep, for it has

Research of the first and second authors supported in part by a grant from the NSF. 
been shown ([Ogg], [Mur]) to be equivalent to the nonvanishing on the line $\operatorname{Re}(s)=1$ of all the $m$ th symmetric power $L$-functions $L_{m}(s)$ associated with $f(z)$.

In this paper we change perspective slightly by fixing $p$ and looking at the distribution of $a_{f}(p)$ as $f(z)$ varies. That is, we are looking at the distribution of the eigenvalues of $T_{p}$.

We write $S_{k}(\Gamma(1))$ for the space of cusp forms of weight $k$ for the full modular group, and we write $f \in S_{k}$ to mean $f \in S_{k}(\Gamma(1))$ and $f$ is a Hecke eigenform. Following Selberg, we write $\sigma_{k}\left(T_{n}\right)$ for the trace of the Hecke operator $T_{n}$ acting on $S_{k}(\Gamma(1))$. Because of our normalization, $\sigma_{k}\left(T_{n}\right)=$ $n^{(k-1) / 2} \sum_{f \in S_{k}} a_{f}(n)$.

Theorem 1. Suppose $p$ is prime. As $k \rightarrow \infty$, the set $\left\{\theta_{f}(p): f \in S_{k}\right\}$ becomes uniformly distributed with respect to the measure

$$
\frac{2}{\pi}\left(1+\frac{1}{p}\right) \frac{\sin ^{2} \theta}{\left(1-\frac{1}{p}\right)^{2}+\frac{4}{p} \sin ^{2} \theta} d \theta .
$$

The same distribution was found by Sarnak [Sar] for the $p$ th coefficient of Maass forms averaged over the Laplacian eigenvalues. Sarnak has pointed out to us that the above measure is the $p$-adic Plancherel measure, and it is also the spectral measure of the nearest-neighbor Laplacian on a $p+1$ regular tree (see $[\mathrm{LPS}]$ ).

Theorem 2. As $p \rightarrow \infty$, with $p$ prime, and $k \rightarrow \infty$ with $k>e^{p}$, the set $\left\{\theta_{f}(p): f \in S_{k}\right\}$ becomes uniformly distributed with respect to the Sato-Tate measure.

A result similar to this was obtained by Birch [B], who considered the distribution of $\theta(p)$ over the elliptic curves over $\mathbb{F}_{p}$. Similar results have also been found for Kloosterman sums ([K], [A], [Mic]) and cubic exponential sums $[\mathrm{L}]$. In each case, results have been obtained by fixing the prime $p$ and varying the other parameters in the problem. The original problem of varying the prime, keeping the other parameters fixed, remains inaccessible.

The idea behind the proofs of the theorems is as follows. Using the Selberg trace formula we obtain a simple expression for $\sum_{f \in S_{k}} a_{f}\left(p^{j}\right)$, valid for large $k$. Using the recurrence relation satisfied by $a_{f}\left(p^{j}\right)$ leads to an expression for $\sum_{f \in S_{k}} a_{f}(p)^{j}$. Such an expression for all $j$ is sufficient to determine the distribution of $\left\{a_{f}(p): f \in S_{k}\right\}$.

2. Initial lemmas. Our starting point is the Selberg trace formula applied to the Hecke operators. The following is formula (4.5) in [Sel]. 
LEMma 1. We have

$$
\begin{aligned}
\sigma_{k}\left(T_{n}\right)= & -\frac{1}{2} \sum_{-2 \sqrt{n}<m<2 \sqrt{n}} H\left(4 n-m^{2}\right) \frac{\eta_{m}^{k-1}-\bar{\eta}_{m}^{k-1}}{\eta_{m}-\bar{\eta}_{m}} \\
& -\sum_{\substack{d \mid n \\
d \leq \sqrt{n}}}^{\prime} d^{k-1}+\delta(\sqrt{n}) \frac{k-1}{12} n^{k / 2-1},
\end{aligned}
$$

where $H(d)$ is the class number, $\delta(x)=1$ if $x$ is an integer and is 0 otherwise, $\sum^{\prime}$ means that a term with $d=\sqrt{n}$ is counted with weight $1 / 2$, and

$$
\eta_{m}=\frac{m+i\left(4 n-m^{2}\right)^{1 / 2}}{2}
$$

The above formula directly leads to an expression for $\sum_{f \in S_{k}} a_{f}\left(p^{j}\right)$. Using the estimate $H(d) \ll d^{1 / 2+\varepsilon}$ gives

Lemma 2. Suppose $p$ is prime. As $k \rightarrow \infty$,

$$
\sum_{f \in S_{k}} a_{f}\left(p^{j}\right)= \begin{cases}\frac{k}{12} \cdot \frac{1}{p^{j / 2}}+O\left(p^{j / 2+\varepsilon}\right), & j \text { even }, \\ O\left(p^{j / 2+\varepsilon}\right), & j \text { odd } .\end{cases}
$$

To make use of the above lemma, it is necessary to relate $a_{f}(p)^{n}$ to $a_{f}\left(p^{j}\right)$. This involves the Chebyshev polynomials $U_{n}(x)$. We have

Lemma 3. Suppose $p$ is prime and write $a_{f}(p)=2 \cos \theta_{f}(p)$. Then

$$
a_{f}\left(p^{j}\right)=U_{j}\left(\cos \theta_{f}(p)\right) \quad \text { and } \quad a_{f}(p)^{n}=\sum_{j=0}^{n} h_{n}(j) a_{f}\left(p^{j}\right),
$$

where

$$
h_{n}(j)=\frac{2^{n+1}}{\pi} \int_{0}^{\pi} \cos ^{n} \theta \sin (j+1) \theta \sin \theta d \theta .
$$

Proof. The first expression follows directly from the recurrence relation $a_{f}\left(p^{j}\right)=a_{f}(p) a_{f}\left(p^{j-1}\right)-a_{f}\left(p^{j-2}\right)$ and the fact that the $U_{j}(x)$ satisfy essentially the same recurrence. To prove the second, note that the $h_{m}(j)$ satisfy

$$
x^{n}=\sum_{j=0}^{n} h_{n}(j) U_{n}(x / 2) .
$$

Since the $U_{n}(x)$ are orthonormal on $[-1,1]$ with respect to the measure $(2 / \pi) \sqrt{1-x^{2}} d x$, we have

$$
h_{n}(j)=\frac{2^{n+1}}{\pi} \int_{-1}^{1} x^{n} U_{n}(x) \sqrt{1-x^{2}} d x .
$$

Changing variables $x \mapsto \cos \theta$ gives the stated formula. 
3. Proof of the theorems. Both theorems follow immediately from this proposition.

Proposition 1. Suppose $p$ is prime. As $k \rightarrow \infty$,

$$
\sum_{f \in S_{k}} a_{f}(p)^{n}=\frac{k}{6 \pi}\left(1+\frac{1}{p}\right) \int_{0}^{\pi} 2^{n} \cos ^{n} \theta \frac{\sin ^{2} \theta}{1+\frac{1}{p^{2}}-\frac{2}{p} \cos 2 \theta} d \theta+O\left(p^{n / 2+\varepsilon}\right) .
$$

Pr o of. We assume $n$ is even, the case of odd $n$ being identical. Starting with the second expression in Lemma 3, and then applying Lemma 2 gives

$$
\begin{aligned}
\sum_{f \in S_{k}} a_{f}(p)^{n} & =\sum_{f \in S_{k}} \sum_{j=0}^{n} h_{n}(j) a_{f}\left(p^{j}\right)=\frac{k}{12} \sum_{j=0}^{n / 2} \frac{h_{n}(2 j)}{p^{j}}+O\left(p^{n / 2+\varepsilon}\right) \\
& =\frac{k}{12} \sum_{j=0}^{\infty} \frac{h_{n}(2 j)}{p^{j}}+O\left(p^{n / 2+\varepsilon}\right)
\end{aligned}
$$

the completion of the sum on $j$ being valid because $h_{n}(j)=0$ for $j>n$. Now inserting the expression for $h_{n}(j)$ from Lemma 3, switching the sum and integral, and then evaluating the sum on $j$ gives

$$
\begin{aligned}
\sum_{f \in S_{k}} a_{f}(p)^{n} & =\frac{k}{6 \pi} \sum_{j=0}^{\infty} \frac{1}{p^{j}} \int_{0}^{\pi} 2^{n} \cos ^{n} \theta \sin (2 j+1) \theta \sin \theta d \theta+O\left(p^{n / 2+\varepsilon}\right) \\
& =\frac{k}{6 \pi} \int_{0}^{\pi} 2^{n} \cos ^{n} \theta \sin \theta \sum_{j=0}^{\infty} \frac{\sin (2 j+1) \theta}{p^{j}} d \theta+O\left(p^{n / 2+\varepsilon}\right) \\
& =\frac{k}{6 \pi}\left(1+\frac{1}{p}\right) \int_{0}^{\pi} 2^{n} \cos ^{n} \theta \frac{\sin ^{2} \theta}{1+\frac{1}{p^{2}}-\frac{2}{p} \cos 2 \theta} d \theta+O\left(p^{n / 2+\varepsilon}\right)
\end{aligned}
$$

as claimed.

The deduction of the theorems from Proposition 1 is by Weyl's criterion: the sequence $\left(b_{n}\right)$ is uniformly distributed on $[a, b]$ with respect to the measure $d \mu$ provided

$$
\lim _{N \rightarrow \infty} \frac{1}{N} \sum_{n=1}^{N} g\left(b_{n}\right)=\int_{a}^{b} g d \mu,
$$

for all $g \in C([a, b])$. Proposition 1 gives the necessary equality for $g(x)=x^{n}$, uniformly in $k$, which is sufficient because the polynomials are dense in $C([a, b])$. This completes the proofs of the theorems. 
Added in proof. We have recently learned that a forthcoming article by J.-P. Serre, to appear in the Journal of the American Mathematical Society, contains similar results.

\section{References}

[A] A. Adolphson, On the distribution of angles of Kloosterman sums, J. Reine Angew. Math. 395 (1989), 214-220.

[B] B. J. Birch, How the number of points of an elliptic curve over a fixed prime field varies, J. London Math. Soc. 43 (1968), 57-60.

[K] N. M. Katz, Gauss Sums, Kloosterman Sums, and Monodromy Groups, Ann. of Math. Stud. 116, Princeton, 1988.

[L] R. Livné, The average distribution of cubic exponential sums, J. Reine Angew. Math. 375/376 (1987), 362-379.

[LPS] A. Lubotzky, R. Phillips and P. Sarnak, Ramanujan graphs, Combinatorica 8 (1988), 261-277.

[Mic] P. Michel, Autour de la conjecture de Sato-Tate pour les sommes de Kloosterman I, Invent. Math. 121 (1995), 61-78.

[Mur] V. K. Murty, On the Sato-Tate conjecture, in: Number Theory Related to Fermat's Last Theorem, Progr. Math. 26, Birkhäuser, Boston, 1981, 195-205.

[Ogg] A. P. Ogg, A remark on the Sato-Tate conjecture, Invent. Math. 9 (1970), 198200.

[Sar] P. Sarnak, Statistical properties of eigenvalues of the Hecke operators, in: Analytic Number Theory and Diophantine Problems (Stillwater, OK, 1984), Progr. Math. 70, Birkhäuser, Boston, 1987, 321-331.

[Sel] A. Selberg, Harmonic analysis and discontinuous groups in weakly symmetric Riemannian spaces with applications to Dirichlet series, J. Indian Math. Soc. B 20 (1956), 47-87; reprinted in: Collected Papers, Vol. I, Springer, Berlin, 1989, 423-463.

[Ser] J.-P. Serre, Abelian l-adic Representations and Elliptic Curves, Benjamin, New York, 1968.

Department of Mathematics

Oklahoma State University

Stillwater, Oklahoma 74078

U.S.A.

E-mail: conrey@math.okstate.edu

Department of Mathematics

Bucknell University

Lewisburg, Pennsylvania 17837

U.S.A.

E-mail: farmer@bucknell.edu
Department of Mathematics Rutgers University New Brunswick, New Jersey 08903 U.S.A.

E-mail: duke@math.rutgers.edu 